\title{
L'éducation à la citoyenneté Françoise Profit
}

\section{OpenEdition}

\section{Journals}

Édition électronique

URL : http://journals.openedition.org/ries/1611

DOI : $10.4000 /$ ries. 1611

ISSN : 2261-4265

Éditeur

Centre international d'études pédagogiques

Édition imprimée

Date de publication : 1 septembre 2003

Pagination : 14-17

ISBN : 978-2-85420-557-1

ISSN : $1254-4590$

Référence électronique

"L'éducation à la citoyenneté Françoise Profit », Revue internationale d'éducation de Sèvres [En ligne],

33 | septembre 2003, mis en ligne le 12 avril 2012, consulté le 19 avril 2019. URL : http://

journals.openedition.org/ries/1611 ; DOI : 10.4000/ries.1611

Ce document a été généré automatiquement le 19 avril 2019.

(C) Tous droits réservés 


\section{L'éducation à la citoyenneté Françoise Profit}

1 Le 7 mai 1999, le comité des ministres du Conseil de l'Europe a adopté la « Déclaration et le programme sur l'éducation à la citoyenneté démocratique fondée sur les droits et les responsabilités des citoyens " ${ }^{1}$, inscrivant ce programme éducatif dans une volonté politique forte. Par cet acte, les instances européennes ont fait prendre conscience à l'ensemble de la communauté internationale, et au sein de chaque pays, de l'enjeu que représente cette question majeure d'éducation. L'expression «éducation à la citoyenneté $~_{2}^{2}$ recouvre plusieurs notions : éducation civique, éducation à l'intégration, à la tolérance, éducation au développement et à la solidarité. Nous avons choisi ici une sélection de sites qui représentent, chacun dans leur domaine, un aspect de cette volonté à l'éducation à la citoyenneté ${ }^{3}$.

2 Après avoir retenu quelques sites qui permettent de cerner la problématique ainsi que les pages qui fournissent les textes de référence, nous abordons une sélection de sites de plusieurs pays qui présentent des actions prospectives et des éléments de recherche sur le sujet. Une dernière partie propose quelques exemples d'applications pédagogiques dans différents pays. Nous ne pouvions être exhaustifs à ce propos, tant la diversité des activités pédagogiques est vaste.

\section{Textes de référence}

\section{Conseil de l'Europe}

3 Les pages consacrées à l'éducation à la citoyenneté démocratique sur le site du Conseil de l'Europe permettent de définir le concept et de cerner les enjeux de cette question éducative.

4 Définition du concept d'éducation à la citoyenneté démocratique $:$ http://www.coe.int/T/ F/Coop \%E9ration_culturelle/-education/E.C.D/ Qu'est ce_que_l'ECD/ 
Ensemble des textes adoptés par le Conseil de l'Europe : http://www.coe.int/T/F/Coop \% E9ration_culturelle/education/E.C.D/-Docu ments_et_publications/Par_type/ Textes_adopt \%E9s/

6 Le site propose également des liens sur les organismes, les organisation nongouvernementales, les réseaux, les organisations gouvernementales internationales et les institutions qui traitent de l'éducation à la citoyenneté : http://www.coe.int/T/F/Coop \% E9ration_culturelle/-education/E.C.D/ Liens_utiles/ ainsi qu'une liste de sites de citoyenneté par ordre alphabétique de pays: http://www.coe.int/T/F/Coop\% E9ration_culturelle/education/E.C.D/Activit\%E9s_au_sein_du_CE/-Projet_1997-2000/ sites.asp

\section{Ministère de la Jeunesse, de l'Éducation et de la Recherche (France)}

7 Le site offre une page très complète recensant les textes qui montrent que l'éducation à la citoyenneté est une thématique éducative transversale: http:// www.eduscol.education.fr/D0090/default.htm

\section{Analyses et perspectives}

8 L'International Association for the Evaluation of Educational Achievement (IEA) a créé dans les années 90 le Civic Education Study (CivEd) qui analyse l'éducation civique dans vingt-huit pays. Le site http://www2.rz.hu-berlin.de/empir_bf/iea_e.html permet de lire les rapports et les contributions de chaque pays ${ }^{4}$.

Dans les Cahiers du service de pédagogie expérimentale de l'université de Liège, Annette Lafontaine et Geneviève Hindryck ont publié « Une enquête internationale sur l'éducation à la citoyenneté : connaissances, représentations, attitudes et engagement des jeunes ». Cette enquête menée sous la direction de l'IEA dresse un état des lieux et examine, dans une perspective comparative, la façon dont les jeunes sont amenés à devenir citoyens. http://www.ulg.ac.be/pedaexpe/pub/cahiers/cahiers7-8/c0078059.pdf

10 Le Système d'information et de recherche sur l'éducation au Canada (SIREC) association canadienne d'éducation, a constitué un dossier sur «l'éducation à la citoyenneté ». Ce dossier, qui s'inscrit dans une perspective historique, présente un état de la recherche, distingue quatre domaines d'action sur le sujet: le domaine civil, le domaine politique, le domaine socio-économique et le domaine culturel ou collectif et décrit les problématiques actuelles. http://www.ceris.ca/f/Citoyen1.html

11 L'association internationale européenne EDIW (Education for an Interdependent World) «a pour but de promouvoir et favoriser des actions et des projets dans le domaine éducatif, d'orienter à la formation de citoyens solidaires et responsables et de stimuler une plus grande justice dans le monde interdépendant ». Elle a organisé en 2000 un séminaire intitulé «Éducation et citoyenneté européenne en milieu scolaire: expériences et méthodologies actives ». Les conférences du séminaire sont disponibles sur le site, dans la langue de l'intervenant : http://www.ediw.org/Cdrom_Education/files/program.htm

12 «Éducation à la citoyenneté dans une perspective planétaire : le cas de la formation initiale et continue du personnel enseignant est le thème du «séminaire des chaires UNESCO francophones » qui s'est tenu en octobre 2000 à Montréal. 

l'intolérance (ECRI) qui offrent des matériels pédagogiques (le manuel Domino en particulier) pour aborder l'éducation à la citoyenneté : http://www.coe.int/T/F/Droits\% 5Fde\%5Fl\%27Homme/Ecri/3\%2DMat\%E9riels\%5Fp\%E9dagogiques/

21 Les clubs UNESCO forment un réseau de partenaires qui permettent d'échanger entre les jeunes et les adultes, de débattre de problèmes de société, en se référant constamment aux droits fondamentaux. http://www.clubs-unesco.asso.fr/. Le site offre le compterendu des actions menées dans les domaines qui touchent la construction du citoyen et permettent un rapprochement entre les peuples. 


\section{Sites nationaux}

\section{Angleterre}

Sur le site des programmes nationaux anglais http://www.nc.uk.net/home.html, outre les programmes concernant l'enseignement à la citoyenneté, on peut consulter des ressources, des exemples de travaux d'élèves et des conseils sur le sujet. http:// www.nc.uk.net/servlets $/$ Subjects?Subject $=\mathrm{Ci}$

Institute for citizenship, association anglaise, promeut la citoyenneté active entre autres à travers des initiatives éducatives pour une participation plus intense à la démocratie. Le site fournit des exemples de ce qui peut être entrepris à tous les niveaux scolaires :

Citizenship education: the global dimension est une association anglaise dont le site portail http://www.citizenship-global.org.uk/ conçu à l'origine pour compléter l'ouvrage du même nom, aborde l'enseignement de l'éducation à la citoyenneté selon les niveaux scolaires. Des ressources en ligne, et des projets sont accessibles auxquels s'ajoute une partie théorique et réflexive qui ancre cet enseignement dans une dimension globale.

\section{Australie}

Discovering Democracy http://www.curri culum.edu.au/democracy/index.htm est un programme australien sur l'éducation à la citoyenneté et l'éducation civique. Une des pages du site fournit un ensemble de ressources pour aider à l'enseignement avec des exemples d'activités de classe : http://www.curriculum.edu.au/democracy/teachciv.htm

\section{Belgique}

L'université de Liège (Belgique) en collaboration avec le Laboratoire de méthodologie de la géographie et le ministère de la Communauté française propose sur son site des pages qui fournissent des «idées fortes pour l'apprentissage de la citoyenneté » et offrent une application en cours de géographie. http://www.ulg.ac.be/geoeco/lmg/competences/ chantier/ethique/eth_democ.html

\section{France}

Le ministère français de la Jeunesse, de l'Éducation et de la Recherche définit ce qu'il entend par « éducation à la citoyenneté » et fournit des exemples d'actions de fond et des exemples d'actions phares : parlement des enfants et concours René Cassin en particulier. http://www.eduscol.education.fr/D0090/default.htm. Le site recense aussi des liens sur les ressources nationales, les ressources académiques et des dossiers thématiques comme « mémoire et histoire » ou " éducation à la défense ».

8 Pour encourager les jeunes à s'intéresser à la citoyenneté, le gouvernement a mis en place un site consacré au droit des jeunes sur lequel se trouvent les informations nécessaires et en particulier un guide thématique pour aider à résoudre des problèmes d'ordre juridique ou social. http://www.droitsdesjeunes.gouv.fr/ 


\section{États-Unis}

29 Le Center for civic education se consacre à l'éducation du citoyen dans le respect de la constitution américaine. Il cherche à développer les programmes internationaux pour le développement de la démocratie à différents niveaux. De nombreux liens, dont une rubrique intitulée " educational resources", sont offerts ainsi que des sites sur les lois et les politiques mises en œuvre : http://www.civiced.org/internet.html

\section{NOTES}

1. Texte disponible à l'adresse suivante: http://www.coe.int/T/F/Coop\%E9ration_culturelle/ education/E.C.D/Documents_et_publications/Par_type/Textes_adopt \%E9s/ decl_et_prog.asp\#TopOfPage

2. Nous avons traduit l'expression "éducation à la citoyenneté » en anglais par Citizenship education ou civic education, en espagnol: educacion a la ciudadania, en allemand: Staatsbürgerkunde et en russe : ОБЩЕСТВЕННОЕ ВоспИТАНИЕ.

3. Malgré nos recherches, nous n'avons pas trouvé de site - récent et de référence - issu du monde hispanophone.

4. Voir la Revue internationale d'éducation de Sèvres, $n^{\circ} 31$, novembre 2002, p. 18, où nous avons déjà présenté ce site à propos des programmes d'évaluations internationales. 